\title{
Neuromodulation devices nowadays
}

\author{
R.E. Rizea ${ }^{1,2}$, Karina Lidia Gheorghita ${ }^{2}$, \\ Gh. David ${ }^{3}$, A.V. Ciurea ${ }^{4}$ \\ 1 Neurosurgery Department, "Bagdasar-Arseni" Emergency Neurosurgery \\ Hospital, Bucharest, RomanIA \\ 2 "Carol Davila" University of Medicine and Pharmacy, Bucharest, RomanIA \\ 3 Neurosurgery Department, "Regina Maria" Military Hospital, Brasov, \\ ROMANIA \\ ${ }^{4}$ Neurosurgery Department, Sanador Hospital, Bucharest, RomanIA
}

\begin{abstract}
Introduction. Neuromodulation devices have known a great progress in the past years being used in treatment of drug resistant neurological diseases such as epilepsies and migraines. A neuromodulation device can stimulate profound or superficial neural pathways in order to balance chronic drug-resistant disorders that involve disturbances of cellular electrical potentials.

Material. Cranial neuromodulation devices implants used until now usually determined skull irregularities, implant site infection, resorption of the bone flap or osteomyelitis. In order to solve these problems, it was needed a customized cranial implant that integrates the neuromodulation device.

We report the first description of a fully integrated neuromodulation device within a customized cranial implant, publicised in 2018 by Gordon et al., that demonstrates the utility of a computerized neurostimulation device combined with clear customdesigned cranial implant.

Conclusion. The new approach of neurotechnology confines a better solution for neuroimplants devices with less follow-up complications and great patient's satisfaction.
\end{abstract}

\section{INTRODUCTION}

Neuromodulation devices stimulate profound or superficial neural pathways in order to balance chronic drug-resistant disorders that involve disturbances of cellular electrical potentials, such as epilepsies and migraines. Most of the cranial neuromodulation device implants used until now had adverse reactions like skull irregularities, implant site infection, resorption of the bone flap or osteomyelitis [1-5].

\section{MATERIAL}

We report the first description of a fully integrated neuromodulation device within a customized cranial implant, publicised in 2018 by Gordon et al., that demonstrates the utility of a computerized neurostimulation device combined with clear custom-designed cranial implant.

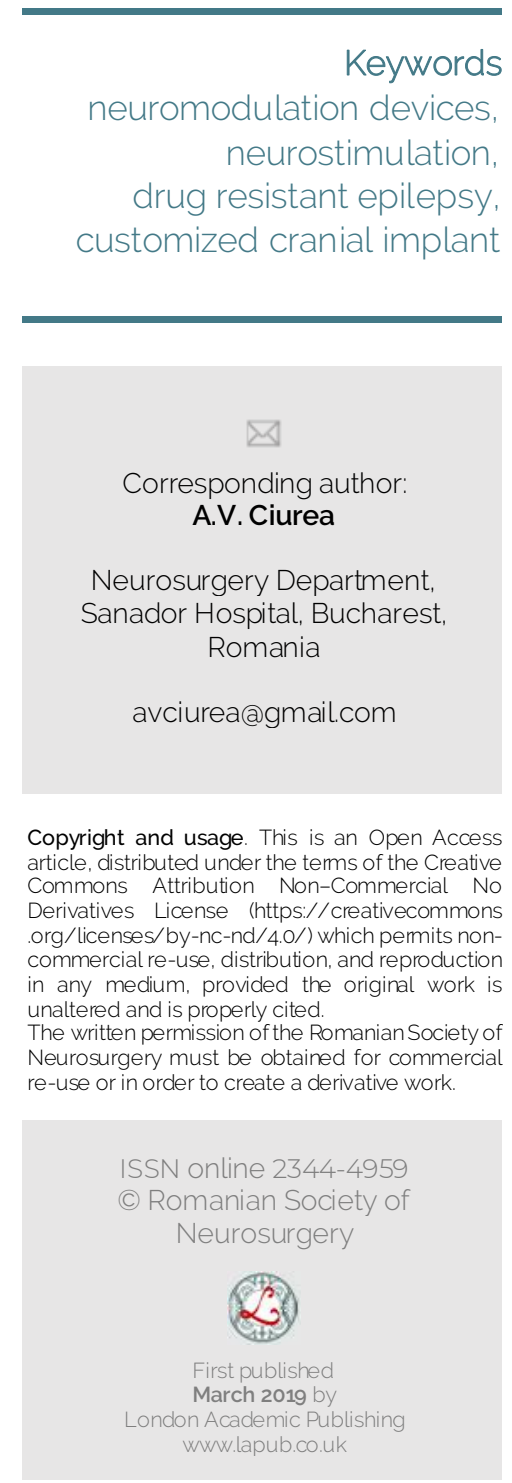


Modern day treatment for drug resistant neurological diseases has known a great progress during past years. In 2018, Gordon et al. from Johns Hopkins Hospital, Maryland, USA, reported first in-human experience using a neuromodulation device within a cranial implant. The neuromodulation was used before in drug resistant epilepsies and migraines $[1,2]$. It is well known that one in three patients with focal seizures develops a drug resistant form of epilepsy. Patients with focal seizures who have failed multiple drug associated treatment that do not fit neurosurgical approach need vagus nerve stimulation or responsive neurostimulation $[1,3,4,5]$.

The responsive neurostimulation system, approved by the FDA in 2013, is used for patients that have drug resistant epilepsy with less than 2 epileptogenic foci and it has a cortical stimulator that detects and respond to the electroencephalographic events $[1,6]$. Even though it has a great efficacy, there has been reported a lot of complications about the montage of the device, such as: scalp dehiscence, device exposure, contamination of hardware, infections, contour irregularities, bone flap osteomyelitis, visual deformities that affect quality of patient's life $[1,7,8]$.

In order to solve all these problems and to reduce the number of re-interventions that increases the risk of infections, Gordon et al. started to plan a new improved implant design. They changed the opaque material used before with a transparent one for a better visibility and accuracy of the positioning to avoid the electrocorticographic signal interference. All the components of the device were integrated below the implant as an incorporated piece, fact that prevents the obvious deformities of the skull and scalp and the migration of the device into the scalp [1, 9-11].

\section{DISCUSSION}

Gordon et al. used a 54 years old drug resistant epilepsy patient who needed responsive neurostimulation device with cranioplasty. A multidisciplinary team formed by neurosurgeons and plastic surgeons produced a perfect size 3D printed mold of the patient's skull that was computer-laser modified with a 5-axis robot laser cutter and after that presterilised $[1,12,13]$. The responsive neurostimulation device with the leads were placed under the clear cranial implant making possible a perfect visualization of the connections and possible inadvertent device interference. The patient was fully recovered with no complications. This was the first description of a fully integrated neuromodulation device within a customized cranial implant $[1,13,14]$.

The method allows the avoidance of visible irregularities, scalp dehiscence, device extrusion and lead migration identified as complications of standard procedures above skull. The technology of cranial implants is expected to develop and treat other brain pathologies such as tumours and movement disorders and maybe it can be implicated more in intelligent cognition devices for memory diseases [1].

\section{CONCLUSION}

We can make a conclusion that the new approach of neurotechnology confines a better solution for neuroimplants devices with less follow-up complications and great patient's satisfaction. It is a great demonstration that can inspire new research in other brain pathologies that can use computerized neurostimulation device combined with clear custom-designed cranial implant $[1,15,16]$.

\section{REFERENCES}

1. Gordon CR, Santiago GF, Huang J, Bergey GK, Liu S., Armand M, Brem H, Anderson WS; First In-Human Experience With Complete Integration of Neuromodulation Device Within a Customized Cranial Implant, Operative Neurosurgery, Volume 15, Issue 1, 1 July 2018, Pages 39-45, https://doi.org/10.1093/ons/opx210

2. Kwan P, Brodie MJ. Early identification of refractory epilepsy. N Engl J Med. 2000;342(5):314-319.

3. Morris GL 3rd, Gloss D, Buchhalter J, Mack KJ, Nickels K, Harden C. Evidence-based guideline update: vagus nerve stimulation for the treatment of epilepsy: report of the Guideline Development Subcommittee of the American Academy of Neurology. Neurology. 2013;81(16):1453-1459.

4. Collins KL, Lehmann EM, Patil PG. Deep brain stimulation for movement disorders. Neurobiol Dis. 2010;38(3):338-345.

5. Fisher RS, Velasco AL. Electrical brain stimulation for epilepsy. Nat Rev Neurol. 2014;10(5):261-270.

6. RNS System in Epilepsy Study GroupMorrell MJ. Responsive cortical stimulation for the treatment of medically intractable partial epilepsy. Neurology. 2011;77(13):1295-1304.

7. Lopez J, Zhong SS, Sankey EW, et al. Time interval reduction for delayed implant-based cranioplasty 
reconstruction in the setting of previous bone flap osteomyelitis. Plast Reconstr Surg. 2016; 137(2): 394e-404e.

8. Berli JU, Thomaier L, Zhong S, et al. Immediate single-stage cranioplasty following calvarial resection for benign and malignant skull neoplasms using customized craniofacial implants. J Craniofac Surg. 2015;26(5):1456-1462.

9. Wei Z, Gordon CR, Bergey GK, Sacks JM, Anderson WS. Implant site infection and bone flap osteomyelitis associated with the NeuroPace responsive neurostimulation system. World Neurosurg. 2016;88(687): e1-e6.

10. Heck CN, King-Stephens D, Massey AD, et al. Twoyear seizure reduction in adults with medically intractable partial onset epilepsy treated with responsive neurostimulation: final results of the RNS System Pivotal trial. Epilepsia. 2014;55(3):432-441.

11. Bergey GK, Morrell MJ, Mizrahi EM, et al. Long-term treatment with responsive brain stimulation in adults with refractory partial seizures. Neurology. 2015;84(8):810-817.
12. Zhong S, Huang GJ, Susarla SM, Swanson EW,Huang J, Gordon CR. Quantitative analysis of dual-purpose, patient-specific craniofacial implants for correction of temporal deformity. Neurosurgery. 2015;11(Suppl 2):220-229; discussion 229.

13. Liu J, Fang J, Murphy R, Gordon CR, Armand M. Design and development of 5-axis cranial implant laser cutting system. In: Proceedings of the ASME 2017 International Design Engineering Technical Conferences \& Computers and Information in Engineering Conference. IDETC/CIE; 2017.

14. Alambeigi F, Sefati S, Murphy R, Armand M, Gordon $C R$, Liu S. A cutting machine for resizing raw implants during surgery. WO 2016 086049. U.S. International Patent application number PCT/US2015/062516. Published February 6, 2016.

15. Anderson WS, Kossoff EH, Bergey GK, Jallo GI. Implantation of a responsive neurostimulator device in patients with refractory epilepsy. Neurosurg Focus. 2008;25(3): E12.

16. Gordon C, Bryndza JR, Basic T. Patient-specific craniofacial implants. Issued Patent 20160081805. Published March 24, 2016. 